Anaesthesist 2020 69:129-136 https://doi.org/10.1007/s00101-019-00643-2 Online publiziert: 2 . September 2019 (c) Der/die Autor(en) 2019

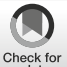

D. Michalski ${ }^{1} \cdot$ C. Jungk ${ }^{2} \cdot$ T. Brenner ${ }^{3} \cdot$ M. Dietrich ${ }^{3} \cdot$ C. Nusshag ${ }^{4} \cdot$ M. A. Weigand ${ }^{3}$. C. J. Reuß $\beta^{3} \cdot$ C. Beynon ${ }^{2} \cdot$ M. Bernhard ${ }^{5}$

' Klinik und Poliklinik für Neurologie, Universitätsklinikum Leipzig, Leipzig, Deutschland

${ }^{2}$ Neurochirurgische Klinik, Universitätsklinikum Heidelberg, Heidelberg, Deutschland

${ }^{3}$ Klinik für Anästhesiologie, Universitätsklinikum Heidelberg, Heidelberg, Deutschland

${ }^{4}$ Klinik für Endokrinologie, Stoffwechsel und klinische Chemie/Sektion Nephrologie, Universitätsklinikum Heidelberg, Heidelberg, Deutschland

${ }^{5}$ Zentrale Notaufnahme, Universitätsklinikum Düsseldorf, Düsseldorf, Deutschland

\title{
Neurologische Intensivmedizin
}

\section{Intensivmedizinische Studien aus 2018/2019}

\section{Intrazerebrale Blutung}

\section{Infobox}

Dieser Beitrag ist Teil einer Serie zu den wichtigsten Intensivmedizinische Studien aus 2018/2019. Weitere Teile der Serie sind:

- Editorial: Coburn M (2019) Intensivmedizinische Studien aus 2018/2019. Anaesthesist. https://doi.org/10.1007/ s00101-019-00638-z

- Flüssigkeitstherapie: Reuß CJ, Dietrich M, Beynon C et al (2019) Flüssigkeitstherapie. Intensivmedizinische Studien aus 2018/2019. Anaesthesist. https://doi.org/ 10.1007/s00101-019-00639-y

- Beatmung und Sauerstofftherapie: Dietrich M, Reuß CJ, Beynon C et al (2019) Beatmung und Sauerstofftherapie. Intensivmedizinische Studien aus 2018/2019. Anaesthesist. https://doi.org/ 10.1007/s00101-019-00640-5

- Fokus Nephrologie: Nusshag C, Beynon C, Dietrich M et al (2019) Fokus Nephrologie. Intensivmedizinische Studien aus 2018/2019. Anaesthesist. https://doi.org/ 10.1007/s00101-019-00641-4

- Additive Therapien: Dietrich M, Reuß CJ, Beynon C et al (2019) Additive Therapien. Intensivmedizinische Studien aus 2018/2019. Anaesthesist. https://doi.org/ 10.1007/s00101-019-00642-3

- Fokus Neurochirurgie: Beynon C, Bernhard M, Brenner T et al (2019) Fokus Neurochirurgie. Intensivmedizinische Studien aus 2018/2019. Anaesthesist. https://doi.org/10.1007/s00101-01900644-1

\section{Originalpublikation \\ Al-Shahi Salman R, Frantzias J, Lee RJ et al (2018) Absolute risk and predictors of the growth of acute spontaneous intracerebral haemorrhage: a systematic review and me- ta-analysis of individual patient data. Lancet Neurol 17:885-894}

Intrazerebrale Blutungen machen innerhalb der Gruppe der Schlaganfälle lediglich etwa ein Fünftel der Fälle aus, jedoch sind diese durch eine vergleichsweise hohe Sterblichkeit bis zu $58 \%$ innerhalb der ersten 12 Monate nach dem Ereignis charakterisiert [1]. Ein besonders hohes Risiko für einen tödlichen Verlauf oder ein schlechtes funktionelles Langzeitergebnis ist assoziiert mit einem im kurzfristigen Behandlungsverlauf nachzuvollziehenden Größenwachstum des Hämatoms [2]. Aktuell fokussieren Therapiestrategien auf eine Vermeidung dieses Größenwachstums, wobei die Blutdruckkontrolle im Fall einer hypertensiven Kreislaufsituation sowie die Herstellung normwertiger Gerinnungsverhältnisse $\mathrm{zu}$ den wesentlichen Therapiezielen gehören. Probleme bestehen hinsichtlich der frühzeitigen Identifizierung von Patienten mit einem potenziell ungünstigen Verlauf.

In einer Metaanalyse unter Einschluss von 77 Studien und insgesamt $5435 \mathrm{~Pa}$ tienten untersuchten Al-Shahi Salmann et al. [3] die Häufigkeit der Größenzunahme von intrakraniellen Hirnblutungen sowie potenziell assoziierten Merkmalen. Eine Größenzunahme der Blutung, definiert als ein Progress von mindestens $6 \mathrm{ml}$, war in 1009 Fällen (20\%) nachvollziehbar. Als unabhängige Prädiktoren für eine Größenzunahme konnten die Einnahme von Thrombozytenaggregationshemmern $(p=0,026)$ oder Antikoagulanzien $(p<0,0001)$, das initiale Blutungsvolumen $(p<0,0001)$ sowie die Zeit zwischen dem Symptombeginn bis zur ersten zerebralen Bildgebung $(p<0,0001)$ identifiziert werden. Das Vorliegen einer CT-Angiographie trug ebenfalls zur Prädiktion bei, da sich das Risiko für eine Größenzunahme der Blutung im Fall eines „spot sign“ (d.h. dem Sichtbarwerden eines Kontrastmittelaustritts innerhalb der Blutung [4]) als Indikator für einen aktiven Zustand) erhöhte. Die Studie von Salman et al. [3] liefert auf der Basis einer umfassenden Stichprobe wertvolle Prädiktoren für einen ungünstigen Verlauf, wodurch Behandlungsinhalte individuell optimiert werden können.

\section{Originalpublikation}

Kuramatsu JB, Sembill JA, Gerner ST et al (2018) Management of therapeutic anticoagulation in patients with intracerebral haemorrhage and mechanical heart valves. Eur Heart J 39: 1709-1723

Intrazerebrale Blutungen stellen v.a. bei Patienten mit mechanischem Herzklappenersatz und sich hieraus ergebender Indikation für eine orale Antikoagula- 
tion eine große Herausforderung dar. Daten sowohl zur Beendigung der oralen Antikoagulation, als auch zu deren Wiederaufnahme nach dem Blutungsereignis waren bislang nicht verfügbar, da die auf Zentrumsebene meist geringen Fallzahlen wissenschaftliche Ansätze mit Handlungsimplikationen häufig verhinderten. Basierend auf einer deutschlandweiten Kohortenstudie unter Beteiligung von 22 Zentren standen die Daten von 2504 Patienten mit einer antikoagulanzienassoziierten intrazerebralen Blutung zur Verfügung. Aus diesem Datensatz konnten die Parameter von 137 Patienten mit einem mechanischen Herzklappenersatz extrahiert werden. Als primärer Endpunkt wurden hämorrhagische Komplikationen erfasst, wie beispielsweise eine Größenzunahme der Hirnblutung um mehr als $33 \%$ oder jegliche neue Hirnblutung. Darüber hinaus wurden thrombembolische Komplikationen vor dem Hintergrund der in der Praxis oft pausierten Antikoagulation berücksichtigt. Von insgesamt $137 \mathrm{~Pa}$ tienten erhielten $71(52 \%)$ im Verlauf keine therapeutische Antikoagulation und 66 Patienten (48\%) eine therapeutische Antikoagulation durch VitaminK-Antagonisten, unfraktioniertes $\mathrm{He}$ parin oder niedermolekulares Heparin in therapeutischer Dosierung. Damit ergaben sich 2 Behandlungsgruppen, die hinsichtlich der Unterbrechung der vorbestehenden Antikoagulation und deren Wiederbeginn analysiert wurden. Im Ergebnis war die inkomplette Normalisierung der Gerinnungssituation assoziiert mit dem erhöhten Risiko für eine Größenzunahme der intrazerebralen Blutung. In der Behandlungsgruppe mit wieder aufgenommener Antikoagulation fanden sich signifikant mehr Blutungskomplikationen (26 vs. $6 \%$; $p<0,01)$ sowie eine tendenziell geringere Anzahl von thrombembolischen Komplikationen ( 2 vs. $10 \% ; p=0,06$ ). Unter Berücksichtigung der relevantesten Komplikationen, konkret des Blutungsprogresses oder anderer Blutungen sowie im Gegensatzhierzu thrombembolischen Komplikationen zeigte sich das beste Wirksamkeits-/Sicherheitsprofil für einen Wiederbeginn der Antikoagulation frühestens ab dem 13. Tag nach dem Ereignis bzw. ab dem 6. Tag bei Patienten mit hohem thrombembolischen Risiko, beispielsweise ausgehend von einem koinzidentellen Vorhofflimmern oder einer Prothese in Mitralposition. Wenngleich das retrospektive Studiendesign die Ableitung einer unmittelbaren Handlungsanweisung für die Praxis verhindert, liefert die Studie von Kuramatsu et al. [5] doch wichtige Erkenntnisse zur Nutzen-Risiko-Bewertung der Antikoagulation bei Patienten mit mechanischem Klappenersatz und eingetretener Hirnblutung, die in der individuellen Therapieplanung Berücksichtigung finden können.

\section{Mechanische Thrombektomie und periprozedurales Management}

\section{Originalpublikation}

Fuhrer $\mathrm{H}$, Schönenberger S, Niesen WD et al (2019) Endovascular stroke treatment's impact on malignant type of edema (ESTIMATE). J Neurol 266:223-231

Die Wirksamkeit endovaskulärer Therapieverfahren und hier im Speziellen der mechanischen Thrombektomie bei der Behandlung akuter, proximaler, intrakranieller Gefäßverschlüsse konnte durch mehrere randomisierte, klinische Studien in den vergangenen Jahren eindrucksvoll belegt werden [6]. Inwieweit die unter kontrollierten Studienbedingungen erreichbaren Ergebnisse sich auch in der Praxis wiederfinden und hier zu einer nachhaltigen Veränderung der Behandlungsqualität führen, war bisher außerhalb von Fallberichten und monozentrischen Kohortenstudien nicht bekannt. In einer multizentrischen Studie untersuchten Fuhrer et al. [7] auf der Basis der zwischen 2007 und 2015 gesammelten Behandlungsdaten aus 5 deutschen Zentren die Auswirkungen der endovaskulären Therapie (mit bzw. auch ohne i.v.-Thrombolyse) gegenüber der alleinigen i.v.-Thrombolyse bzw. keiner Akutintervention bei Patienten mit akutem Hirninfarkt - basierend auf einem Verschluss der A. carotis interna oder der A. cerebri media. Primärer
Endpunkt war die Entwicklung eines ausgedehnten Hirninfarkts. Darüber hinaus wurden u.a. die Sterblichkeit und das funktionelle Behandlungsergebnis betrachtet. Einbezogen wurden die Daten von 2161 Patienten, von denen 691 $(32,1 \%)$ einen malignen Hirninfarkt entwickelten, im Wesentlichen definiert als eine Einbeziehung von mehr als $66 \%$ des Versorgungsgebiets der A. cerebri media. Bezogen auf die angewandten Therapiestrategien war die endovaskuläre Therapie dabei signifikant assoziiert $(p<0,01)$ mit dem geringsten Anteil an Patienten mit ebendieser Infarktentwicklung $(23,8 \%)$ im Vergleich zur systemischen Thrombolyse (32,2\%) und der fehlenden Akuttherapie (50,1\%). Vergleichbare Ergebnisse fanden sich hinsichtlich der Sterblichkeit und des funktionellen Behandlungsergebnisses: Die endovaskuläre Therapie war mit der geringsten Rate an intrahospitaler Sterblichkeit (9,6 vs. 19,8 bzw. $28,1 \%$ ) und dem höchsten Anteil von Patienten mit einem günstigen funktionellen Ergebnis, d.h. einer modifizierten RankinSkala (mRS) von 0 bis 3, assoziiert (22,1 vs. 9,3 bzw. $5,7 \% ; p<0,01)$. Die Studie von Fuhrer et al. [7] bestätigt damit die Effektivität der endovaskulären Therapie bei Patienten mit proximalem Gefäßverschluss auch auf der Versorgungsebene. Weiterführende Untersuchungen zur geänderten Versorgungslandschaft nach dem Jahr 2015, in dem die mechanische Thrombektomie der Leitlinie zur Behandlung des Hirninfarkts der Deutschen Gesellschaft für Neurologie [8] hinzugefügt wurde, werden sicher folgen.

\section{Originalpublikation}

Campbell BCV, Majoie CBLM, Albers GW et al (2019) Penumbral imaging and functional outcome in patients with anterior circulation ischaemic stroke treated with endovascular thrombectomy versus medical therapy: a meta-analysis of individual patient-level data. Lancet Neurol 18:46-55

Im Jahr 2018 konnten 2 randomisierte, klinische Studien einen positiven Effekt der mechanischen Thrombektomie auf das funktionelle Behandlungsergebnis auch bei einer Durchführung im erwei- 
terten Zeitfenster, konkret bis 16h [9] bzw. 24 h [10] nach dem Symptombeginn, zeigen. Bedingt durch diese Ergebnisse wurde eine deutliche Änderung der Behandlungspraxis erwartet, da nach theoretischen Überlegungen weitaus mehr Patienten für eine Akuttherapie beim Hirninfarkt infrage kommen würden als bisher. Aus medizinischen und logistischen Gesichtspunkten erschienen Kriterien wünschenswert, die anhand bildgebender Parameter eine Vorhersage des Behandlungsergebnisses erlauben und somit beispielsweise Sekundärverlegungen in Zentren mit vorgehaltener endovaskulärer Therapie auch unter Inkaufnahme eines für den Patienten möglichen Risikos durch den Transport rechtfertigen. Eine im Jahr 2019 veröffentlichte Studie von Campbell et al. [11] untersuchte in Form einer Metaanalyse unter Zuhilfenahme der 7 verfügbaren, randomisierten, klinischen Studien im Bereich der mechanischen Thrombektomie die Beziehung zwischen der initialen, multimodalen zerebralen Bildgebung (CT oder MRT) und dem funktionellen Behandlungsergebnis nach 3 Monaten. Hierbei fanden die Daten von 1764 Patienten Berücksichtigung und zeigten die Zunahme des ischämischen Kerngebiets, im Wesentlichen definiert als relativer zerebraler Blutfluss von weniger als $30 \%$, in Schritten von $10 \mathrm{ml}$ assoziiert mit einer jeweils 20 - bis $30 \%$ igen Reduktion der Wahrscheinlichkeit für eine funktionelle Unabhängigkeit (mRS 0-2) nach dem Ereignis $(p<0,0001)$. Weiterführende Analysen ergaben, dass ein klinisch relevanter Vorteil bis zu einem Volumen von $150 \mathrm{ml}$ des Infarktkerns nachvollziehbar war. Signifikante Einflussfaktoren waren zudem das Patientenalter, das Ausmaß der neurologischen Defizite und die Zeit bis zur Rekanalisation. Diese umfangreiche Sekundäranalyse von Campbell et al. [11] liefert robuste Hinweise, dass mithilfe der multimodalen zerebralen Bildgebung sowie einzelner zusätzlicher Merkmale Patienten identifiziert werden können, die auch im erweiterten Zeitfenster von einer endovaskulären Therapie profitieren und somit entsprechenden Zentren zugeführt werden sollten. Dass ein Teil der Patienten selbst außerhalb des bisher untersuchten 24- stündigen Zeitfensters eine perfusionsgestützte zerebrale Bildgebung aufweist, die theoretisch einer Intervention zugänglich wäre, wurde in einer anderen Untersuchung kürzlich berichtet [12]; therapeutische Implikationen ergeben sich zum jetzigen Zeitpunkt hieraus jedoch noch nicht.

\section{Originalpublikation}

Pandhi A, Tsivgoulis G, Ishfaq MF et al (2019) Mechanical thrombectomy outcomes in large vessel stroke with high international normalized ratio. J Neurol Sci 396:193-198

Mithilfe der oralen Antikoagulation als Sekundärprophylaxe nach kardioembolisch bedingtem Hirninfarkt kann das Risiko eines weiteren Ereignisses reduziert werden. Dennoch ergeben sich Situationen, in denen ein Hirninfarkt unter einer bestehenden Antikoagulation auftritt und dieser im Fall der Einnahme eines Vitamin-K-Antagonisten und einer INR $>1,7$ mit der systemischen Thrombolyse aufgrund des assoziierten Blutungsrisikos nicht behandelt werden kann. Wenige Daten lagen bisher für die mechanische Thrombektomie unter diesen Bedingungen vor. In einer bizentrischen Studie untersuchten Pandhi et al. [13] 315 konsekutive, mit der mechanischen Thrombektomie behandelte Patienten, von denen 10 eine INR >1,7 aufwiesen. Diese Gruppe unterschied sich gegenüber den übrigen Patienten nicht hinsichtlich des Auftretens von symptomatischen intrakraniellen Blutungen $(p=0,706)$, der Sterblichkeit $(p=0,762)$ und der Rate an Patienten mit funktioneller Unabhängigkeit (mRS 0-2) nach 3 Monaten. Diese Ergebnisse konnten anhand einer Metaanalyse aufder Basis von 5 bereits vorhandenen Kohortenstudien mit insgesamt 80 antikoagulierten Patienten (INR > 1,7) und 1545 Kontrollen (INR <1,7) bestätigt werden. Unter Berücksichtigung des rein deskriptiven Designs liefert die Studie von Pandhi et al. [13] das für die Praxis relevante Ergebnis, dass die mechanische Thrombektomie bei Patienten mit erhöhter INR sicher zu sein scheint und somit im Fall eines intrakraniellen, proximalen Gefäßverschlusses eine kausale Behandlungsmöglichkeit darstellt.

\section{Originalpublikation}

Pfaff JAR, Schönenberger $S$, Nagel $S$ et al (2018) Effect of general anesthesia versus conscious sedation for stroke thrombectomy on angiographic workflow in a randomized trial: a post hoc analysis of the SIESTA trial. Radiology 286:1016-1021

Hinsichtlich des periprozeduralen Managements der mechanischen Thrombektomie lieferte die zurückliegende Arbeit von Brinjikji et al. [14] den Hinweis, dass die alleinige Sedierung der Allgemeinanästhesie im Hinblick auf das funktionelle Behandlungsergebnis überlegen ist. Die 3 vorhandenen, prospektiven, randomisierten Studien konnten diesen Effekt nicht bestätigen und zeigten im Bereich der sekundären Endpunkte teilweise sogar eine Überlegenheit der Allgemeinanästhesie gegenüber der alleinigen Sedierung [15-17]. In einer Sekundäranalyse der Studie "Sedation versus Intubation for Endovascular Stroke Treatment" (SIESTA) fokussierten Pfaff et al. [18] auf den Effekt von Allgemeinanästhesie und Sedierung auf den Arbeitsablauf während der mechanischen Thrombektomie und hier speziell auf die benötigte Zeit zwischen der Leistenpunktion und der Rekanalisation des Gefäßverschlusses. Zur Verfügung standen die Daten von 77 sedierten und 73 Patienten mit Allgemeinanästhesie, wobei periprozedural 11 Patienten von einer primär randomisierten Sedierung in eine Allgemeinanästhesie überführt wurden. Zwischen den beiden Behandlungsgruppen fanden sich weder relevante Unterschiede hinsichtlich der Zeitdauer zwischen der Leistenpunktion und der angiographischen Darstellung des Gefäßverschlusses ( 16 vs. $15 \mathrm{~min} ; p=0,396$ ) noch bezüglich der Zeit zwischen der Leistenpunktion und dem ersten Manöver, das zu einer Wiederherstellung des intrakraniellen Blutflusses führte (47 vs. $41 \mathrm{~min} ; p=0,546$ ). Die Allgemeinanästhesie war im Gegensatz zur Sedierung sogar mit einer kürzeren Zeit zwischen der Leistenpunktion und der finalen angiographischen Abbildung assoziiert, d.h. dem Zeitpunkt, der sich an das individuell letzte Rekanalisationsmanö- 
ver noch vor Abschluss der Prozedur anschließt (71 vs. $98 \mathrm{~min} ; p=0,048$ ). In Ergänzung zu den vorhandenen prospektiven, randomisierten Untersuchungen mit zumindest neutralem Effekt von Allgemeinanästhesie und Sedierung auf das funktionelle Behandlungsergebnis, erweitert die Studie von Pfaff et al. [18] die Diskussion hinsichtlich des optimalen periprozeduralen Managements um neuroradiologische Prozessabläufe und zeigt einen möglichen positiven Effekt der Allgemeinanästhesie auf. Der mögliche Nettogewinn der Allgemeinanästhesie im Vergleich zur alleinigen Sedierung muss in randomisierten Studien mit großer Fallzahl verifiziert werden, sodass zum jetzigen Zeitpunkt ein heterogenes Gesamtbild verbleibt, das die Wahl des Verfahrens auf Fallebene weiter an individuelle Bedingungen knüpft.

\section{Originalpublikation}

Rasmussen M, Espelund US, Juul N et al (2018) The influence of blood pressure management on neurological outcome in endovascular therapy for acute ischaemic stroke. $\mathrm{Br} J$ Anaesth 120:1287-1294

Neben der grundsätzlichen Frage des periprozeduralen Verfahrens hinsichtlich der alleinigen Sedierung bzw. der Allgemeinanästhesie existieren derzeit wenige Daten zu den hämodynamischen Auswirkungen dieser beiden Vorgehensweisen. Frühere Diskussionen beinhalteten v. a. die Möglichkeit der narkosebedingten Hypotension mit negativen Effekten auf das funktionelle Langzeitergebnis. In einer Sekundäranalyse der Studie „General or Local Anesthesia in Intra Arterial Therapy“ (GOLIATH), die 128 Patienten in die Gruppen Allgemeinanästhesie vs. Sedierung randomisierte [17], untersuchten Rasmussen et al. [19] die hämodynamischen Auswirkungen beider Verfahren und deren Beziehung zum Behandlungsergebnis. Das Protokoll der GOLIATH-Studie sah periprozedural einen systolischen Zielblutdruck von mehr als $140 \mathrm{~mm} \mathrm{Hg}$ und einen mittleren arteriellen Blutdruck von mehr als $70 \mathrm{~mm} \mathrm{Hg}$ vor. Im Ergebnis fanden sich höhere mittlere systolische und mittlere arterielle Blutdruckwerte in der Behand- lungsgruppe mit alleiniger Sedierung $(p<0,001)$. Mithin war der Anteil der Patienten mit reduzierten Blutdruckwerten und Vasopressorbedarfin der Gruppe der Allgemeinanästhesie signifikant erhöht $(p<0,001)$. Eine signifikante Beziehung zwischen den erfassten Blutdruckwerten und -abweichungen sowie dem funktionellen Behandlungsergebnis, gemessen mit der mRS nach 3 Monaten, bestand dagegen nicht. Auch unter Berücksichtigung der sich durch das retrospektive Studiendesign ergebenden Limitationen zeigt die Studie von Rasmussen et al. [19], dass die beiden aktuell verfügbaren periprozeduralen Verfahren mit hämodynamischen Auswirkungen einhergehen, wenngleich eine unmittelbare Beziehung zum funktionellen Ergebnis hieraus jedoch nicht zwangsläufig abzuleiten ist. Das optimale Blutdruckmanagement vor, während und nach der mechanischen Thrombektomie zählt auch weiterhin zu den offenen Fragen im Bereich der endovaskulären Behandlung von Hirninfarkten [20]. Um Einblicke in mögliche Kausalzusammenhänge zu erzielen, sind diesbezüglich kontrollierte, randomisierte Studien notwendig.

\section{Raumfordernder Hirninfarkt}

\section{Originalpublikation}

Gul W, Fuller HR, Wright H et al (2018) A systematic review and meta-analysis of the effectiveness of surgical decompression in treating patients with malignant middle cerebral artery infarction. World Neurosurg 120: e902-920

Der raumfordernde, maligne Hirninfarkt als Maximalvariante der fokalen zerebralen Ischämie geht mit einer Sterblichkeit bis zu $80 \%$ einher [21]. In den vergangenen ca. 10 Jahren konnten randomisierte, klinische Studien die Wirksamkeit der dekompressiven Hemikraniektomie belegen [22]. Kontrovers diskutiert wurden jedoch die gewählten primären Endpunkte, die vorrangig auf eine Reduktion der Sterblichkeit fokussierten und Überlebende oft durch schwere neurologische Folgezustände charakterisiert waren, wenngleich sich der Großteil der Patienten mit dem Behandlungsergebnis zufrieden zeigte [23]. Gerade für die klinische Praxis und hier die Beratung Betroffener und deren Familien erschienen weiterführende Analysen notwendig, die Daten zur Effektivität der dekompressiven Hemikraniektomie über den Faktor Sterblichkeit hinaus liefern und somit als Entscheidungshilfe für die individuelle Therapieplanung herangezogen werden können. In einer Metaanalyse untersuchten Gul et al. [24] 18 Studien, von denen die Hälfte randomisiert war. Die Autoren analysierten auf Grundlage der Daten von 987 Patienten die nach der dekompressiven Hemikraniektomie bestehenden funktionellen Einschränkungen 3, 6, 12 und 36 Monate nach dem Ereignis und vollzogen zudem die Sterblichkeitsraten an ebendiesen Zeitpunkten nach. Ein Überlebensvorteil durch die dekompressive Hemikraniektomie konnte dabei unabhängig vom Alter gezeigt werden, wenn der Eingriff innerhalb von $48 \mathrm{~h}$ nach dem Beginn des Schlaganfalls durchgeführt wurde. Dieser Vorteil zeigte sich konsistent über alle Zeitpunkte, d.h. bis 36 Monate nach dem Ereignis ( $p$-Werte zwischen $<0,00001$ und 0,01). Getrennt betrachtet von der Sterblichkeit fanden sich im Vergleich der Hemikraniektomie gegenüber der Kontrollgruppe, die der besten Therapie ohne Hemikraniektomie entsprach, keine signifikanten Unterschiede in der Häufigkeit eines ungünstigen $(m R S=5)$ gegenüber einem günstigeren ( $\mathrm{mRS}<5$ ) Ergebnis während der gesamten Beobachtungszeit. Ungeachtet der schwierigen Definition eines günstigen Behandlungsergebnisses und hier im Speziellen der gewählten Dichotomisierung im Übergang der mRS 4 zu 5 bestätigt die Metaanalyse von Gul et al. [24] die Wirksamkeit der dekompressiven Hemikraniektomie bei der Behandlung des malignen Hirninfarkts hinsichtlich der Sterblichkeit auch im Langzeitverlauf. Soweit angesichts des Studiendesigns zulässig, zeigt die Studie aber auch den - im Fall eines Überlebens - fehlenden Einfluss der dekompressiven Hemikraniektomie auf den funktionellen Zustand. Umso mehr ergibt sich hieraus die Notwendigkeit einer individualisierten Therapieplanung, für die patienteneigene Wertevorstellungen meist im Gespräch mit Angehörigen ermittelt 
und mit dem erreichbaren Behandlungsergebnis abgeglichen werden sollten.

\section{Originalpublikation}

Kimberly WT, Bevers MB, von Kummer R et al (2018) Effect of IV glyburide on adjudicated edema endpoints in the GAMES-RP trial. Neurology 91:e2163-2169

Neben der dekompressiven Hemikraniektomie wurden in den vergangenen Jahren medikamentöse und damit weniger invasive Ansätze zur Behandlung des malignen Hirninfarkts untersucht. Im Jahr 2016 wurde eine multizentrische, randomisierte, doppelblinde, klinische Studie veröffentlicht, in der die Sicherheit und Wirksamkeit des Antidiabetikums Glibenclamid bei Patienten mit ausgedehntem Hirninfarkt untersucht werden sollten [25]. Vorangegangen waren Beobachtungen aus tierexperimentellen Untersuchungen, die eine Reduktion des schlaganfallbedingten Hirnödems zeigten. Die Studie wurde nach dem Einschluss von 86 Patienten aus finanziellen Gründen abgebrochen; es ergaben sich durch die Glibenclamid- gegenüber der Placebogabe keine Unterschiede hinsichtlich des primären Endpunkts, konkret des Anteils der Patienten mit mRS 0-4 ohne Hemikraniektomie nach 3 Monaten. Eine im Jahr 2018 veröffentlichte Sekundäranalyse dieser Studie untersuchte nun den Einfluss von Glibenclamid auf die Sterblichkeit und weitere Parameter speziell bei Patienten mit einer malignen Ödementwicklung, die definiert war als: National Institutes of Health Stroke Scale (NIHSS) >18, reduzierte Vigilanz und raumfordernder Infarkt in der zerebralen Bildgebung [26]. Hierbei zeigte sich am Tag 30 nach dem Hirninfarkt eine Reduktion der Sterblichkeit durch Gabe von Glibenclamid (2,4 vs. $8,22 \% ; p=0,010$ ), wobei dieser Effekt noch deutlicher wurde bei Patienten mit einer malignen Ödementwicklung $(p=0,009)$. In der Subpopulation mit maligner Ödementwicklung war die Gabe von Glibenclamid mit einer geringeren Mittellinienverlagerung (4,6 vs. $12,4 \mathrm{~mm} ; p=0,001)$ und einem geringeren neurologischen Defizit, gemessen mit der NIHSS innerhalb der ersten
3 Tage $(p=0,001)$, assoziiert. Wenngleich es sich um eine Sekundäranalyse mit entsprechenden Limitationen handelt, so deutet die Studie von Kimberly et al. [26] auf einen ödemreduzierenden Effekt von Glibenclamid hin, der zukünftig genutzt werden könnte, um ein verbessertes Behandlungsergebnis des malignen Hirninfarkts zu erzielen. Weiterführende und v.a. prospektiv angelegte Studien unter Einsatz dieser Substanz und mit einem primären Effektivitätsendpunkt bleiben derzeit jedoch abzuwarten.

\section{Hypothermie}

\section{Originalpublikation}

van der Worp HB, Macleod MR, Bath PMW et al (2019) Therapeutic hypothermia for acute ischaemic stroke. Results of a European multicentre, randomised, phase III clinical trial. Eur Stroke J Apr 20. https://doi.org/10.1177/ 2396987319844690

Für die induzierte Hypothermie mit einer Zieltemperatur von 32 bis $34^{\circ} \mathrm{C}$ konnten in randomisierten klinischen Studien für den konvulsiven Status epilepticus [27] und die bakterielle Meningitis [28] keine überzeugenden Effekte hinsichtlich des funktionellen Ergebnisses nach 3 Monaten erzielt werden. Es fanden sich dagegen kritische Signale hinsichtlich der Sicherheit der Hypothermie in diesem Anwendungsbereich, da eine erhöhte Sterblichkeit bzw. eine vermehrte Rate an Komplikationen (z.B. Pneumonie) nachvollziehbar waren [28]. Unsicherheit bestand bisher hinsichtlich der Wirkung der induzierten Hypothermie auf die fokale zerebrale Ischämie, d.h. den Hirninfarkt, wobei die Ergebnisse aus tierexperimentellen Studien und theoretische Überlegungen zahlreiche positive Effekte nahelegten [29]. Mit Spannung erwartet wurden daher die Ergebnisse der internationalen, multizentrischen, randomisierten, kontrollierten, endpunktverblindeten EuroHYP-1-Studie, die - gefördert durch die Europäische Union - eine innerhalb von $6 \mathrm{~h}$ nach Symptombeginn induzierte und für einen Zeitraum von 12 bzw. 24 h aufrechterhaltene Hypothermie mit $34-35^{\circ} \mathrm{C}$ bei Patienten mit Hirnin- farkt im Vergleich zur Standardbehandlung untersuchte [30]. Zum Einsatz kamen sowohl Oberflächenkühlsysteme als auch endovaskuläre Verfahren. Als primärer Endpunkt wurde der funktionelle Zustand, bewertet mit der mRS am Tag 91 nach dem Hirninfarkt definiert, wobei als gutes Ergebnis eine mRS 0-2 angesehen wurde. Als Stichprobengröße waren 1500 Patienten vorgesehen. Die Rekrutierung startete im November 2013 und wurde nach 98 Patienten (Hypothermie: $n=49$, Kontrolle: $n=49$; eingeschlossen in 23 Zentren) im März 2018 aufgrund der geringen Rekrutierungsrate und der endenden Förderperiode eingestellt. Die systemische Thrombolyse wurde während der Standardbehandlung in beiden Gruppen nahezu gleich häufig angewandt (39 vs. 41 Patienten). In der Interventionsgruppe erreichten lediglich rund $50 \%$ der Patienten die geplante Dauer der Hypothermie. Als Grund wurdev. a. ein schwer kontrollierbares ,shivering" der per se nichtsedierten Patienten berichtet, wenngleich die Studie ein spezielles Anti-Shivering-Protokoll mit u.a. einer maximalen Dosis von $500 \mathrm{mg} \mathrm{Pe}$ thidin beinhaltete. Hinsichtlich des primären Endpunkts fanden sich keine Unterschiede zwischen den Behandlungsgruppen (OR 1,$01 ; p=0,97$ ), ebenso wie hinsichtlich der Sterblichkeit (RR 1,25; $p=0,73$ ) und des rein neurologischen $\mathrm{Zu}$ stands, bewertet mit der NIHSS (Median 3 vs. $3 ; p=0,39$ ). Die Anzahl an schwerwiegenden unerwünschten Ereignissen unterschied sich zwischen den Behandlungsgruppen nicht (RR 1,22; $p=0,52$ ), wenngleich die Rate an Pneumonien numerisch in der Hypothermiegruppe erhöht war ( $n=9$ vs. $n=2)$. Durch die Studie von van der Worp et al. [30] reiht sich der Hirninfarkt neben solchen neurologischen Erkrankungen ein, bei denen sich die aus theoretischen Überlegungen abgeleiteten positiven Effekte der induzierten Hypothermie unter klinischen Bedingungen nicht nachvollziehen ließen. Sicher wären mit einer weit größeren Gruppengröße nachhaltigere Aussagen zum Effekt der Hypothermie möglich gewesen, jedoch verdeutlicht die Studie durch die Rekrutierungszahl die assoziierten Schwierigkeiten gerade bei wachen Patienten und erhärtet durch eine 
möglicherweise erhöhte Pneumonierate das zuvor bereits vorhandene Signal eines nicht unkritischen Sicherheitsprofils der Hypothermie.

\section{Originalpublikation}

Neugebauer $\mathrm{H}$, Schneider $\mathrm{H}$, Bösel J et al (2019) Outcomes of hypothermia in addition to decompressive hemicraniectomy in treatment of malignant middle cerebral artery stroke: a randomized clinical trial. JAMA Neurol Jan 18. https://doi.org/10.1001/jamaneurol. 2018.4822

Trotz der in den vergangenen Jahren erzielten, v.a. im Zusammenhang mit der dekompressiven Hemikraniektomie stehenden Fortschritte bei der Behandlung des malignen Hirninfarkts verläuft die Erkrankung auch bei Ausschöpfung der therapeutischen Möglichkeiten in ca. $20 \%$ der Fälle tödlich [31]. Vor dem Hintergrund potenzieller neuroprotektiver Effekte und einer damit möglich erscheinenden Reduktion der Sterblichkeit erwuchs in den vergangenen Jahren die Überlegung, die induzierte Hypothermie als additive Therapiestrategie zur medikamentösen und zur chirurgischen Behandlung des malignen Hirninfarkts einzusetzen, die in einer klinischen Studie mündete [32]. In Form einer multizentrischen, randomisierten, kontrollierten, endpunktverblindeten Studie untersuchten Neugebauer et al. [33] nun den Effekt einer frühzeitig nach der Hemikraniektomie induzierten und für eine Dauer von $72 \mathrm{~h}$ aufrechterhaltenen Hypothermie mit $33^{\circ} \mathrm{C}$ gegenüber der Standardbehandlung bei Patienten mit malignem Hirninfarkt. Vorgesehen war der Einschluss von 324 Patienten. Die Rekrutierung startete im August 2011 und wurde im September 2015 nach Einschluss von 50 Patienten (Hypothermie: $n=26$, Kontrolle: $n=24$; eingeschlossen in 6 Zentren) aus Sicherheitsgründen beendet. Der Zielkorridor hinsichtlich Körpertemperatur und Dauer der Hypothermie wurde von $88 \%$ der Patienten in der Hypothermiegruppe erreicht. Als primären Endpunkt beinhaltete die Studie die Sterblichkeitsrate innerhalb von 14 Tagen, die sich zwischen der Hypothermie- und der Kontrollgruppe nicht unterschied (19 vs. $13 \%$; $p=0,70$ ). Auch fanden sich zwischen den beiden Behandlungsgruppen keine Unterschiede hinsichtlich des Ausmaßes der neurologischen Defizite nach 2 Wochen und des funktionellen Zustands nach einem Jahr, erfasst mit NIHSS und mRS. Nachdem sich der Anteil von Patienten mit schwerwiegenden unerwünschten Ereignissen innerhalb des ersten, 14-tägigen Beobachtungszeitraums zwischen den Behandlungsgruppen ohne signifikanten Unterschied zeigte (46 vs. $29 \%$; $p=0,26$ ), wies die Hypothermiegruppe innerhalb des einjährigen Beobachtungszeitraums mit $80 \%$ signifikant mehr Ereignisse gegenüber der Kontrollgruppe mit nur $43 \%$ auf $(p=0,005)$. Die Rate an Pneumonien unterschied sich dabei jedoch nicht zwischen den Behandlungsgruppen (54 vs. $63 \%$; $p=0,74$ ). Die Studie von Neugebauer et al. [33] belegt in Ergänzung zur Untersuchung von van der Worp et al. [30], dass sich für die induzierte Hypothermie auch beim malignen Hirninfarkt die bestehenden theoretischen Vorteile nicht in der klinischen Situation abbilden lassen. Vor dem Hintergrund, dass in der Frühphase des malignen Hirninfarkts zumeist eine Sedierung vorhanden ist und somit ein Shivering deutlich besser kontrollierbar erscheint, bekräftigen die Ergebnisse von Neugebauer et al. [33] zudem das nicht unkritische Sicherheitsprofil der Hypothermie, auch unabhängig vom zu erwartenden Shivering beim wachen Patienten. Ob die induzierte Hypothermie im Einzelfall als Ultima-RatioAnsatz bei sonst nicht kontrollierbarem Hirndruck eine sinnvolle Option darstellt, kann durch die aktuelle Studie nicht beantwortet werden. Hierzu wären weiterführende, klinische Untersuchungen wünschenswert, die aus ethischen Aspekten vermutlich nicht in einem randomisierten Setting durchführbar wären, sodass deren Zustandekommen unwahrscheinlich erscheint.

\section{Status epilepticus}

\section{Originalpublikation}

Madžar D, Reindl C, Giede-Jeppe A et al (2018) Impact of timing of continuous intravenous anesthetic drug treatment on outcome in refractory status epilepticus. Crit Care 22:317

In ca. $25 \%$ der Fälle entwickelt sich ein Status epilepticus zu einem (therapie-) refraktären Status epilepticus [34]. Neben der eigentlichen Statusbehandlung können sich im weiteren Verlauf zudem intensivmedizinische Problemstellungen pulmonaler sowie kardialer Art ergeben, die einen relevanten Einfluss auf das Behandlungsergebnis haben [35]. Im Fall eines refraktären Status epilepticus unter bereits erfolgter Benzodiazepingabe und der etablierten spezifischen antiepileptischen Therapie mit z.B. Phenytoin, Valproat oder Levetiracetam, ergibt sich die Möglichkeit der Eskalation der Statusbehandlung durch den Einsatz von i.v.-Narkosemitteln [36, 37]. Daten zu optimalem Zeitpunkt und Umfang der Eskalation sowie deren Beziehungen zum funktionellen Behandlungsergebnis waren bislang nur ansatzweise verfügbar. In einer retrospektiven, monozentrischen Studie untersuchten Madžar et al. [38] unter den Bedingungen einer Intensivstation den frühen $(<48 \mathrm{~h}) v s$. späteren Einsatz von i.v.-Narkosemitteln (Midazolam, Propofol, Thiopental oder Ketamin) beim (therapie-)refraktären Status epilepticus. Im Behandlungszeitraum zwischen Januar 2001 und Januar 2015 konnten 131 Patienten mit 159 Episoden eines (therapie-)refraktären Status epilepticus identifiziert werden, wobei 83 Episoden mit i.v.-Narkosemitteln behandelt wurden. Für die finale Auswertung standen Daten von 77 Patienten zur Verfügung, von denen 53 (68,8\%) frühzeitig behandelt wurden. Der Großteil der Patienten $(76,6 \%)$ erhielt bis $\mathrm{zu}$ 2 Narkosemittel, teilweise war aber auch der Einsatz aller 4 untersuchten Substanzen notwendig. In 85,7 \% der Fälle gelang eine Terminierung des Status epilepticus. Im Ergebnis war die frühzeitige Eskalation durch die Anwendung von i.v.Narkosemitteln ein unabhängiger Prä- 
diktor für ein gutes Behandlungsergebnis $(p=0,013)$, definiert als eine $\mathrm{mRS}<3$ bzw. eine dem Ausgangsniveau entsprechende mRS. Auch war in der Gruppe mit frühzeitiger Eskalation gegenüber der Kontrollgruppe eine frühzeitigere Terminierung des (therapie-)refraktären Status epilepticus nachvollziehbar ( $\mathrm{HR}=1,796 ; p=0,033)$, und es zeigte sich ein Trend hin zu einer kürzeren Beatmungsdauer in ebendieser Behandlungsgruppe ( $p=0,061)$. Wenngleich das retrospektive und unizentrische Studiendesign zahlreiche Limitationen mit sich bringt, liefert die Untersuchung von Madžar et al. [38] den Hinweis, dass Patienten mit einem (therapie-)refraktären Status epilepticus von einer frühzeitigen Eskalation profitieren könnten. Wünschenswert sind nun weiterführende, prospektiv angelegte Untersuchungen, die sich mit speziellen Aspekten wie der Auswahl und der Dosis der Substanzen innerhalb der Stufe der bereits eskalierten Behandlung des Status epilepticus beschäftigen.

\section{Zusammenfassung: neuro- logische Intensivmedizin}

Im Bereich der neurologischen Akutund Intensivmedizin konnten durch neue klinische Studien Faktoren identifiziert werden, die bei Patienten mit intrazerebralen Blutungen mit einem ungünstigen Behandlungsverlauf assoziiert sind und somit frühzeitig helfen, individualisierte Therapiekonzepte $\mathrm{zu}$ erarbeiten. Die mechanische Thrombektomie konnte als Standardtherapie für die Behandlung des Schlaganfalls mit proximalem Gefäßverschluss und weiteren spezifischen Charakteristika etabliert werden. Neue Untersuchungen ermöglichen anhand der multimodalen zerebralen Bildgebung die zunehmend präzisere Identifizierung von Patienten, die von dieser Therapieform auch in einem erweiterten Zeitfenster profitieren könnten. Erkenntnisse mit in der Zukunft sicher therapeutischen Implikationen waren auch hinsichtlich der Optimierung des periprozeduralen Managements der mechanischen Thrombektomie und der Anwendung dieser Therapie bei bestimmten Risikokonstel- lationen, wie der oralen Antikoagulation, zu verzeichnen. Bei der Behandlung des malignen Hirninfarkts konnten weiterführende Analysen bestätigen, dass die dekompressive Hemikraniektomie einsetzbar ist, um die Sterblichkeit signifikant zu reduzieren, ohne jedoch eine funktionelle Verbesserung zu erzielen. Diese Erkenntnisse sollten bei der Therapieplanung vor dem Hintergrund individueller Wertevorstellungen und Lebensinhalte berücksichtigt werden. Ob medikamentöse, auf eine reduzierte Ödementwicklung ausgerichtete, Therapieansätze bei der Behandlung des malignen Hirninfarkts einen nachhaltigen Effekt zeigen, wird in randomisierten Studien zu untersuchen sein. Für die induzierte Hypothermie liefern 2 aktuelle klinische Studien überzeugende Ergebnisse, dass deren Einsatz beim Hirninfarkt und insbesondere beim malignen Hirninfarkt nicht zu einem verbesserten Behandlungsergebnis führt.

\section{Korrespondenzadresse}

\section{Prof. Dr. med. D. Michalski}

Klinik und Poliklinik für Neurologie, Universitätsklinikum Leipzig Liebigstraße 20, Haus 4, 04103 Leipzig, Deutschland dominik.michalski@medizin.uni-leipzig.de

Interessenkonflikt. D. Michalski, C. Jungk, T. Brenner, M. Dietrich, C. Nusshag, M.A. Weigand, C.J. Reuß, C. Beynon und M. Bernhard geben an, dass kein Interessenkonflikt besteht.

Open Access. Dieser Artikel wird unter der Creative Commons Namensnennung 4.0 International Lizenz (http://creativecommons.org/licenses/by/4.0/deed. de) veröffentlicht, welche die Nutzung, Vervielfältigung, Bearbeitung, Verbreitung und Wiedergabe in jeglichem Medium und Format erlaubt, sofern Sie den/die ursprünglichen Autor(en) und die Quelle ordnungsgemäßnennen, einen Linkzur Creative Commons Lizenz beifügen und angeben, ob Änderungen vorgenommen wurden.

\section{Literatur}

1. Kolominsky-Rabas PL, Heuschmann PU (2002) Incidence, etiology and long-term prognosis of stroke. Fortschr Neurol Psychiatr 70(12):657-662

2. Davis SM et al (2006) Hematoma growth is a determinant of mortality and poor outcome after intracerebral hemorrhage. Baillieres Clin Neurol 66(8):1175-1181

3. Al-Shahi Salman R et al (2018) Absolute risk and predictors of the growth of acute spontaneous intracerebral haemorrhage: a systematic review and meta-analysis of individual patient data. Lancet Neurol 17(10):885-894

4. Rodriguez-Luna D et al (2016) Ultraearly hematoma growth in active intracerebral hemorrhage. Baillieres Clin Neurol 87(4):357-364

5. Kuramatsu JB et al (2018) Management of therapeutic anticoagulation in patients with intracerebral haemorrhage and mechanical heart valves. Eur Heart J39(19):1709-1723

6. Ganesh A, Goyal M (2018) Thrombectomy for acute Ischemic stroke: recent insights and future directions. Curr Neurol Neurosci Rep 18(9):59

7. Fuhrer $\mathrm{H}$ et al (2019) Endovascular stroke treatment's impact on malignant type of edema (ESTIMATE). J Neurol 266(1):223-231

8. Ringleb PA et al (2015) S2k-Leitlinie Akuttherapie des ischämischen Schlaganfalls - Ergänzung 2015; Rekanalisierende Therapie. Leitlinien für Diagnostik und Therapie in der Neurologie, Bd. 2015

9. Albers GW et al (2018) Thrombectomy for stroke at 6 to 16 hours with selection by perfusion imaging. NEngl J Med 378(8):708-718

10. Nogueira RG et al (2018) Thrombectomy 6 to 24 hours after stroke with a mismatch between deficit and infarct. NEngl J Med 378(1):11-21

11. Campbell BCV et al (2019) Penumbral imaging and functional outcome in patients with anterior circulation ischaemic stroke treated with endovascular thrombectomy versus medical therapy: a metaanalysis of individual patient-level data. Lancet Neurol 18(1):46-55

12. Christensen $S$ et al (2019) Persistent target mismatch profile $>24$ hours after stroke onset in DEFUSE 3. Stroke 50(3):754-757

13. Pandhi A et al (2019) Mechanical thrombectomy outcomes in large vessel stroke with high international normalized ratio. J Neurol Sci 396:193-198

14. Brinjikji W et al (2017) Anesthesia-related outcomes for Endovascular stroke Revascularization: a systematic review and meta-analysis. Stroke 48(10):2784-2791

15. Lowhagen Henden P et al (2017) General anesthesia versus conscious sedation for Endovascular treatment of acute Ischemic stroke: the anstroke trial (anesthesia during stroke). Stroke 48(6):1601-1607

16. Schonenberger $S$ et al (2016) Effect of conscious sedation vs general anesthesia on early neurological improvement among patients with Ischemic stroke undergoing Endovascular Thrombectomy: a randomized clinical trial.JAMA 316(19):1986-1996

17. Simonsen CZ et al (2018) Effect of general anesthesia and conscious sedation during Endovascular therapy on infarct growth and clinical outcomes in acute Ischemic stroke: a randomized clinical trial. Jama Neurol 7(5):470-477

18. Pfaff JAR et al (2018) Effect of general anesthesia versus conscious sedation for stroke Thrombectomy on Angiographic Workflow in a randomized trial: a post hoc analysis of the SIESTA trial. Radiology 286(3):1016-1021

19. Rasmussen M et al (2018) The influence of blood pressure management on neurological outcome in endovascular therapy for acute ischaemic stroke. BrJ Anaesth 120(6):1287-1294

20. Jadhav AP et al (2018) Care of the postThrombectomy patient. Stroke 49(11):2801-2807

21. Huttner HB, Schwab S (2009) Malignant middle cerebral artery infarction: clinical characteristics, treatment strategies, and future perspectives. Lancet Neurol 8(10):949-958 
22. Juttler E et al (2014) Hemicraniectomy in older patients with extensive middle-cerebral-artery stroke. NEngl J Med 370(12):1091-1100

23. Rahme $R$ et al (2012) Decompressive hemicraniectomy for malignant middle cerebral artery territory infarction: is life worth living? J Neurosurg 117(4):749-754

24. Gul W et al (2018) A systematic review and meta-analysis of the effectiveness of surgical decompression in treating patients with malignant middle cerebral artery infarction. World Neurosurg 120:e902-e920

25. Sheth KN et al (2016) Safety and efficacy of intravenous glyburide on brain swelling after large hemispheric infarction (GAMES-RP): a randomised, double-blind, placebo-controlled phase 2 trial. Lancet Neurol 15(11):1160-1169

26. Kimberly WT et al (2018) Effect of IV glyburide on adjudicated edema endpoints in the GAMES-RP Trial. Baillieres Clin Neurol 91(23):e2163-e2169

27. Legriel $S$ et al (2016) Hypothermia for Neuroprotection in convulsive status Epilepticus. N Engl J Med 375(25):2457-2467

28. Mourvillier B et al (2013) Induced hypothermia in severe bacterial meningitis: a randomized clinical trial. JAMA 310(20):2174-2183

29. Froehler MT, Ovbiagele B (2010) Therapeutic hypothermia for acute ischemic stroke. Expert Rev Cardiovasc Ther 8(4):593-603

30. van der Worp HB et al (2019) Therapeutic hypothermia for acute ischaemic stroke. Results of a European multicentre, randomised, phase III clinical trial. Eur Stroke J. https://doi.org/10.1177/ 2396987319844690

31. Neugebauer H, Juttler E (2014) Hemicraniectomy for malignant middle cerebral artery infarction: current status and future directions. Int J Stroke 9(4):460-467

32. Neugebauer $\mathrm{H}$ et al (2013) DEcompressive surgery Plus hypoTHermia for Space-Occupying Stroke (DEPTH-SOS): a protocol of a multicenter randomized controlled clinical trial and a literature review. Int JStroke 8(5):383-387

33. Neugebauer $H$, Schneider $H$, Bösel J, Hobohm C, Poli S, Kollmar R, Sobesky J, Wolf S, Bauer M Tittel S, Beyersmann J, Woitzik J, Heuschmann PU, Jüttler E (2019) Outcomes of hypothermia in addition to decompressive hemicraniectomy in treatment of malignant middle cerebral artery stroke: a randomized clinical trial. JAMA Neuro 76(5):571-579

34. Jayalakshmi S et al (2015) Aetiology and outcome of generalized convulsive status epilepticus in elderly. Seizure 29:104-108

35. Sutter R et al (2018) Acute systemic complications of convulsive status Epilepticus-A systematic review. Crit Care Med 46(1):138-145

36. Betjemann JP, Lowenstein DH (2015) Status epilepticus in adults. Lancet Neurol 14(6):615-624

37. Rosenow F et al (2012) S2k-Leitlinie Status epilepticus im Erwachsenenalter. Leitlinien für Diagnostikund Therapie in der Neurologie

38. Madzar D et al (2018) Impact of timing of continuousintravenousanestheticdrug treatment on outcome in refractory status epilepticus. Crit Care 22(1):317

\section{Erratum}

Anaesthesist 2020 -69:136

https://doi.org/10.1007/s00101-020-00733-6

Online publiziert: 29. Januar 2020

(c) Springer Medizin Verlag GmbH, ein Teil von Springer Nature 2019

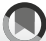

Check for
updates

\section{Neuhaus ${ }^{1}$ - E. Fella² - G. Breuer ${ }^{2}$}

${ }^{1}$ Klinik für Anästhesiologie, AG Patientensicherheit, \& Simulation, Universitätsklinikum Heidelberg, Heidelberg, Deutschland

${ }^{2}$ Klinik für Anästhesiologie und Operative Intensivmedizin, Regiomed Klinikum Coburg, Lehrkrankenhaus der Universität Split, Coburg, Deutschland

\section{Erratum zu: 76/m Totalendoprothese der Hüfte. Vorbereitung auf die Facharztprüfung: Fall 32}

\section{Erratum zu:}

Anaesthesist 2019

https://doi.org/10.1007/s00101-019-

00696-3

In dem ursprünglichen Artikel wurde der Name der Autorin E. Fella falsch angegeben. Bitte beachten Sie die korrigierte Schreibweise.

\section{Korrespondenzadresse}

\section{Dr. C. Neuhaus, M.Sc.}

Klinik für Anästhesiologie, AG Patientensicherheit, \& Simulation, Universitätsklinikum Heidelberg Im Neuenheimer Feld 110, 69120 Heidelberg, Deutschland christopher.neuhaus@med.uni-heidelberg.de
Die Online-Version des Originalartikels ist unter https://doi.org/10.1007/s00101-019-00696-3 zufinden. 